\title{
Mizoribine provides effective treatment of sequential histological change of arteritis and reduction of inflammatory cytokines and chemokines in an animal model of Kawasaki disease
}

Kei Takahashi ${ }^{1}$, Toshiaki Oharaseki ${ }^{1}$, Tomokazu Nagao ${ }^{2}$, Yuki Yokouchi ${ }^{1}$, Hitomi Yamada ${ }^{1}$, Noriko Nagi-Miura ${ }^{3}$, Naohito Ohno ${ }^{3}$, Tsutomu Saji ${ }^{4}$, Tomio Okazaki ${ }^{5}$ and Kazuo Suzuki ${ }^{*}$

\begin{abstract}
Background: Intravenous immunoglobulin (IVlg) treatment results in an effective response from patients with acute-phase Kawasaki disease (KD), but $16.5 \%$ of them remain nonresponsive to IVlg. To address this therapeutic challenge, we tried a new therapeutic drug, mizoribine (MZR), in a mouse model of KD, which we have established using injections of Candida albicans water-soluble fractions (CAWS).
\end{abstract}

Methods: CAWS (4 mg/mouse) were injected intraperitoneally into C57BL/6N mice for 5 consecutive days. MZR or IgG was administered for 5 days. After 4 weeks, the mice were sacrificed and autopsied, the hearts were fixed in $10 \%$ neutral formalin, and plasma was taken to measure cytokines and chemokines using the Bio-Plex system. The incidence of panvasculitis in the coronary arteries and aortic root was 100\% in the control group. The incidence of panvasculitis in the MZR group decreased to 50\%. Moreover, the scope and severity of the inflammation of those sites were significantly reduced in the MZR group as well as the IgG group. On the other hand, increased cytokines and chemokines, such as IL-1 $\alpha$, TNF- $\alpha$, KC, MIP-1 $\alpha$, GM-CSF, and IL-13, in the nontreatment group were significantly suppressed by treatment with MZR, but the MCP-1 level increased. In addition, IL-1 $\alpha$, TNF- $\alpha, \mathrm{IL}-10, \mathrm{LL}-13$, and MIP-1 $\alpha$ were suppressed by treatment in the IgG group.

Results: The incidence of panvasculitis in the coronary arteries and aortic root was 100\% in the control group. The incidence of panvasculitis in the MZR group decreased to 50\%. Moreover, the scope and severity of the inflammation of those sites were significantly reduced in the MZR group as well as the IgG group. On the other hand, increased cytokines and chemokines, such as IL-1 $\alpha$ TNF- $\alpha$, KC, MIP-1 $\alpha$, GM-CSF, and IL-13, in the nontreatment group were significantly suppressed by treatment with MZR, but the MCP-1 level increased. In addition, IL-1 $\alpha$, TNF- $\alpha, \mathrm{IL}-10, \mathrm{LL}-13$, and MIP-1 $\alpha$ were suppressed by treatment in the IgG group.

Conclusion: MZR treatment suppressed not only the incidence, range, and degree of vasculitis, but also inflammatory cytokines and chemokines in the plasma of the KD vasculitis model mice, suggesting that MZR may be useful for treatment of KD.

Keywords: Kawasaki disease, an animal model, IVlg, coronary arteritis, inflammatory cytokines and chemokines, mizoribine

\footnotetext{
* Correspondence: ksuzuki@faculty.chiba-u.jp

${ }^{2}$ Inflammation Program, Dept. of Immunology, Chiba University Graduate

School of Medicine, Chuo-ku, Chiba, 260-8670, Japan

Full list of author information is available at the end of the article
} 


\section{Background}

Kawasaki disease (KD) is an acute febrile illness that manifests mainly in infancy and early childhood [1]. The most important complication of KD is coronary arteritis, which leads to formation of aneurysms. KD has attracted special interest because it may cause ischemic heart disease in children due to thrombosed coronary aneurysms [2]. Since the etiology and development of $\mathrm{KD}$ are thought to be due to the dysfunction of the immune system, intravenous immunoglobulin (IVIg) during the early acute phase has been used with an excellent response in most patients [3]. However, 16.5\% of patients did not respond to the first IVIg treatment [4], and some nonresponders to the first IVIg treatment manifested severe coronary arteritis with large aneurysm [5]. Therefore, additional treatments have been tried on the nonresponders to the first treatment with IVIg. To date, a second IVIg treatment [6], plasmapheresis [7-10], pulse steroids [11], cyclophosphamide plus steroids [12], ulinastatin as an elastase inhibitor [13-16], cyclosporin A plus steroids and methotrexate plus steroids [17,18], and anti-tumor necrosis factor- $\alpha$ (infliximab) therapy [19-23] have been tried. Thus, for treatment of patients with KD who do not respond to IVIg, other medicines for immune response and suppression of lymphocyte proliferation have been applied due to immune dysfunction in the patients. One immune modulating medicine, mizoribine (MZR), a drug that inhibits synthesis of purine compounds (GMP), blocks proliferation of lymphocytes and will be useful for application to nonresponders to IVIg treatment. MZR has long been used as therapy for kidney transplantation, lupus nephritis, nephrotic syndrome, and rheumatoid disease with few side-effects [24]. Moreover, it has been reported to have been used for lupus nephritis, nephrotic syndrome, and IgA nephritis in children [25-28], and as a maintenance therapy in anti-neutrophil cytoplasmic autoantibody (ANCA)-associated renal failure, frequently relapsing nephrotic syndrome, and purpura nephritis $[29,30]$. Therefore, MZR will be a valuable therapeutic strategy for patients with KD who are nonresponsive to IVIg.

Prior to a clinical trial in children with $K D$, it was necessary to test MZR in a mouse model of KD, which has been established. The model we chose was the mouse model in which coronary arteritis can be induced by administration of Candida albicans water-soluble fractions (CAWS) [31]. This model mouse has previously been useful for evaluation of other drug treatments.

Therefore, in the present study, we tested MZR as a immunomodular for treatment of this CAWS-induced coronary arteritis. The evaluation of MZR was performed by histopathological findings and profiles of chemokines and cytokines. Also, this treatment effect was compared with that of IgG.

\section{Methods}

\section{Animals}

Four-week-old male C57BL/6N mice were purchased from Charles River Japan (Yokohama, Japan). All mice were kept under specific pathogen-free (SPF) conditions, according to the guidelines for animal care of the National Institute of Infectious Diseases in Tokyo (NIID).

\section{Preparation of CAWS}

CAWS was prepared from C. albicans strain IFO1385 in accordance with the reported method [31]. Briefly, 5 liters of medium (C-limiting medium) was added to a glass incubator, and the culture was maintained for 2 days at $27^{\circ} \mathrm{C}$ while air was supplied at a rate of 5 liters/ $\mathrm{min}$ and the mixture was swirled at $400 \mathrm{rpm}$. Following culture, an equal volume of ethanol was added. After allowing this to stand overnight, the precipitate was collected. After dissolving the precipitate in $250 \mathrm{ml}$ of distilled water, ethanol was added and the mixture was allowed to stand overnight. The precipitate was collected and dried with acetone to obtain CAWS.

\section{Administration of MZR and IgG to the mice}

CAWS ( $4 \mathrm{mg} / \mathrm{mouse} /$ day) in a volume of $0.2 \mathrm{ml}$ was intraperitoneally injected into a C57BL/6N mouse (4week old male) on each of 5 consecutive days. Subsequently, MZR (a kind gift of Asahikasei Pharma Corporation (Tokyo, Japan)) was administered at a dose of $30 \mathrm{mg} / \mathrm{kg} /$ day intraperitoneally for 5 days from the third day of CAWS injection (MZB group), according to the schecule for treatments such as IgG for patients with $\mathrm{KD}$, and the dosage as described elsewhere [32]. Mice for the control group were intraperitoneally treated with $0.2 \mathrm{ml}$ of Dulbecco's phosphate-buffered saline (PBS). After 35 days, the mice were sacrificed by carbon dioxide asphyxiation; autopsy was performed to obtain plasma, and hearts were fixed with $10 \%$ neutralized formalin. For a positive control, treatment with intraperitoneal human IgG (Kenketsu Glovenin I, a kind gift of Nihon Pharmaceutical Co. Ltd., Tokyo, Japan) was performed at a dose of $400 \mathrm{mg} / \mathrm{mouse} /$ day, or for a negative control saline containing $0.1 \%$ glucose (SG) was injected for 5 days according to the same procedures as described elsewhere (IgG group) [33]. The start date of the drug administration was based on the results that the administration from the third experimental day had been the most effective to suppress the development of vasculitis. 


\section{Histological evaluation}

The fixed hearts were embedded in paraffin and sectioned. To observe the histological changes in the coronary arteries and the aorta in detail, 20 to 30 horizontal step sections per mouse were made every $20 \mu \mathrm{m}$. Hematoxylin and eosin (H\&E)-stained sections were prepared by using routine techniques for examination by light microscopy [31]. First, we investigated the incidence of mice with panvasculitis in each group. Panvasculitis was defined as inflammation of all layers of the walls of the coronary arteries and/or the aorta. Then, for quantitative evaluation of vascular inflammation, we divided the area of the aortic root and coronary arteries into five segments and graded the intensity of inflammation in each segment as follows: score 3, panvasculitis; score 2, inflammation involving the tunica intima and adventitia; score 1, inflammation localized to the tunica intima; and score 0, no inflammatory cell infiltration in the vascular wall. A section with the severe inflammation was observed in each segment. The scope of inflammation was defined as the number of segments evaluated as score 1 or more in each mouse, and the severity of the arteritis was defined as the average score of the five segments in each mouse.

Measurement of cytokines and chemokines with Bio-Plex Cytokines and chemokines in the plasma of mice autopsied were measured by a Bio-Plex system. An aliquot of serum $(12 \mu \mathrm{l})$ collected from peripheral blood and diluted 4-fold with the dilution solution was measured for concentration of cytokines by the 23-Plex kit using Bio-Plex 200 according to the manufacturer's protocol and analyzed by the Bio-Plex Luminex 100 XYP instrument (Bio-Rad, Hercules, California, USA). We assayed the following 23 cytokines and chemokines: IL-1 $\alpha$, IL$1 \beta$, IL-2, IL-3, IL-4, IL-5, IL-6, IL-9, IL-10, IL-12p40, IL12p70, IL-13, IL-17, eotaxin, G-CSF, GM-CSF, INF- $\gamma$, KC, MCP-1, MIP-1 $\alpha$, MIP- $1 \beta$, RANTES, and TNF- $\alpha$ as estimated with a single assay to a single standard curve described in the kit instructions. Concentrations of cytokines and chemokines were calculated using Bio-Plex Manager 3.0 software (Bio-Rad, Tokyo) with a five-parameter curve-fitting algorithm applied for standard curve calculations [34].

\section{Statistical analysis}

Fisher's exact probability test was used to analyze the differences in the incidence of arteritis among the groups. The data on the scope and severity of the arteritis and cytokine/chemokine levels were analyzed using the two-sample $t$-test. A value of $P<0.05$ was considered statistically significant.

\section{Results}

Histological evaluation of panvasculitis in treatment with MZR

Panvasculitis developed in the coronary arteries and the aortic root, and histology was similar to that previously described [33]. Specifically, vascular changes were classified as proliferative inflammation that consisted mainly of large mononuclear cells such as histiocytes and fibroblasts and of a small number of neutrophils. The normal structure of the arteries was completely destroyed, and the internal elastic lamina, external elastic lamina, and smooth muscle layer of the tunica media were severely damaged. However, fibrinoid necrosis was not observed in any of the mice. In addition, the histology of panvasculitis was similar in the three groups (Figure 1).

\section{Decrease of coronary arteritis by treatment with MZR}

Panvasculitis of the coronary arteries and the aortic root was observed in 5 of 5 mice $(100 \%)$ in the nontreated control group. On the other hand, the incidence of panvasculitis in the MZR group was 3 of 6 mice (50\%), and the IgG group as an effective control showed 3 of 7 (43\%) (Figure 2A). In addition, the number of segments evaluated as score 1 or more in each MZR group was decreased compared with the nontreated control group $(P=0.06)$, and the scope of inflammation in IgG groups was significantly lower than in the control group $(P<$ 0.05 ) (Figure 2B). Furthermore, the severity of the arteritis, i.e., the scores of each of five segments in the mice in the MZR and IgG groups, was significantly lower than in the nontreated control group $(P<0.01)$ (Figure 2C).

\section{Reduction of inflammatory cytokines and chemokines by treatment with MZR and IgG}

Inflammatory cytokines IL-1 $\alpha$, TNF- $\alpha$, chemokines KC, MIP-1 $\alpha$, GM-CSF, and Th2, and cytokine IL-13 in plasma of mice, which were inoculated with CAWS in the control group, were elevated (Figure 3). However, in the MZR group, plasma levels of inflammatory cytokines IL- $1 \alpha(P<0.01)$ and TNF- $\alpha(P<0.05)$, and chemokines KC $(P<0.01)$, MIP-1 $\alpha(P<0.01)$, and GM-CSF $(P<$ $0.05)$ were significantly suppressed (Figure $3 \mathrm{~A})$. Inversely, the MCP-1 level increased with MZR treatment (Figure 3A). On the other hand, IL-1 $\alpha(P<0.05)$, TNF$\alpha(P<0.05)$, IL-10 $(P<0.05)$, and IL-13 $(P<0.01)$ were suppressed by administration of IgG (Figure $3 \mathrm{~B}$ ).

Furthermore, we analyzed levels of cytokines/chemokines in plasma, which were related with suppression of the development of coronary arteritis by treatment with MZR. As shown in Figure 4A, the suppression levels were almost the same in all plasmas of MZR-treated mice. These results are not the same as those in the IgG 


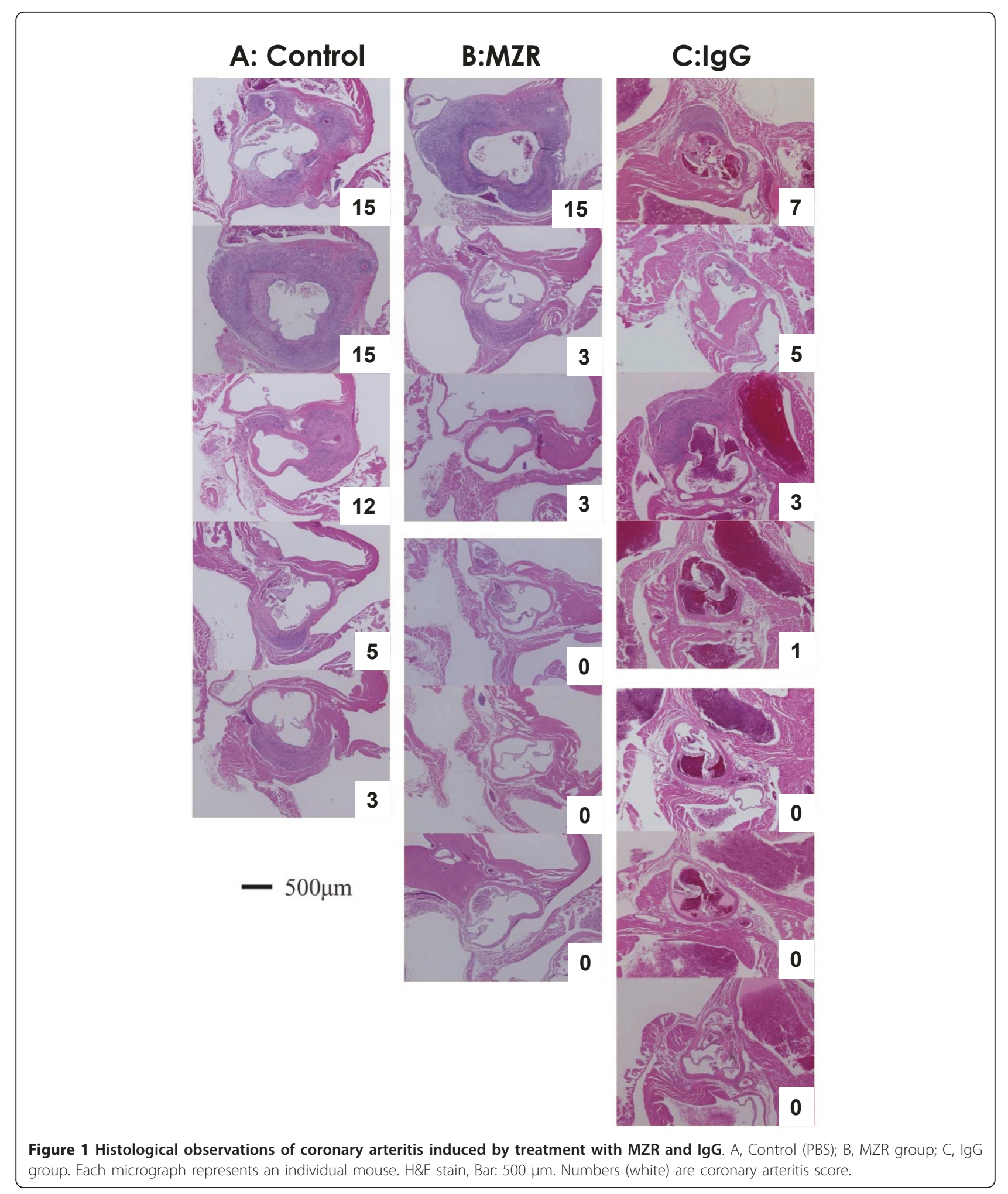




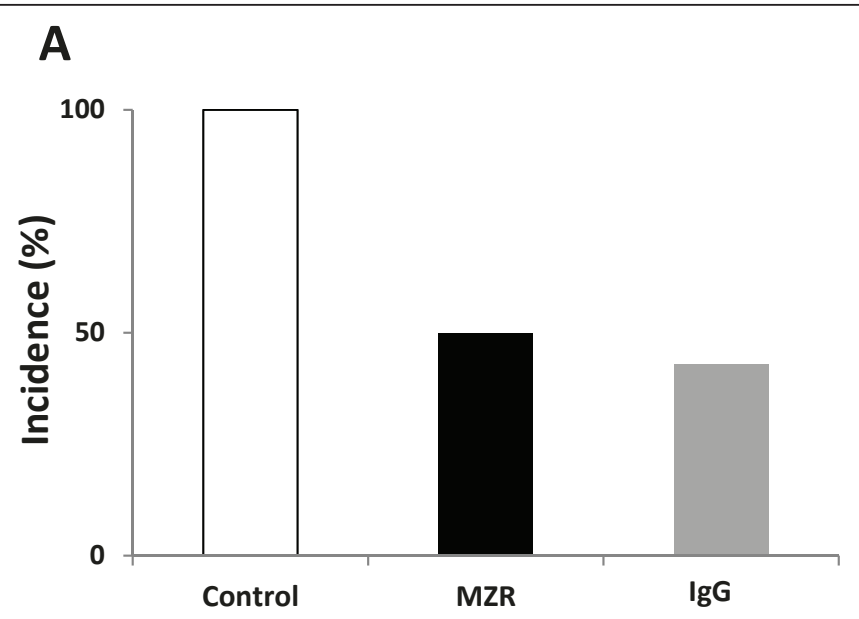

B

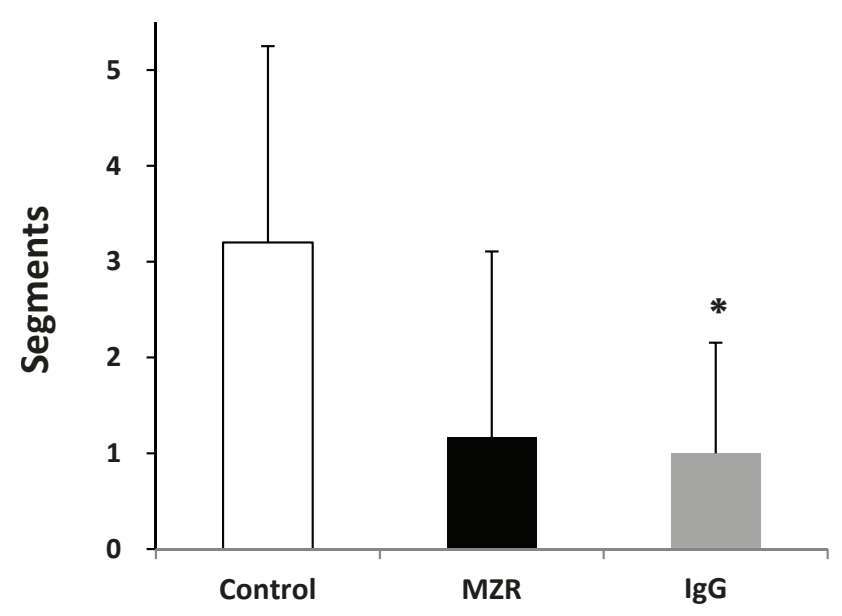

C

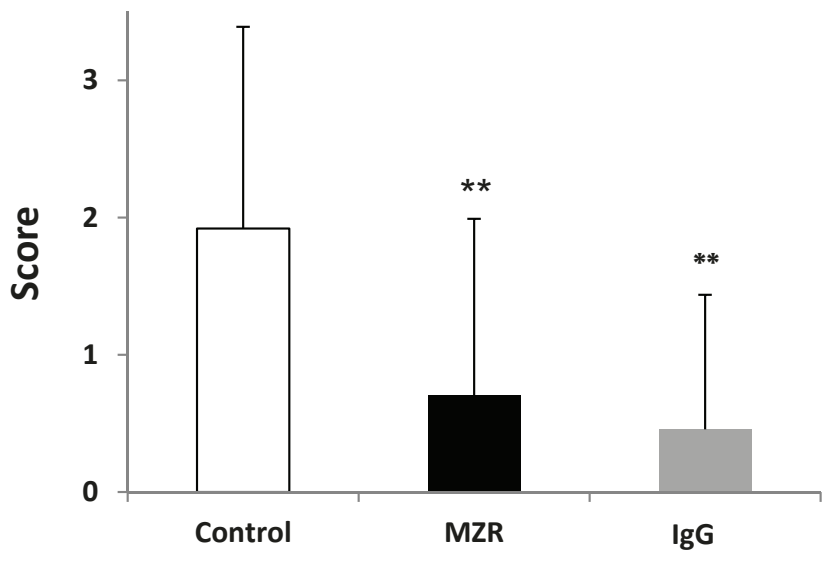

Figure 2 Decrease of incidence of panvasculitis, segment score, and severity score of coronary arteritis by treatment with MZR and IgG. A, incidence of development of panvasculitis; B, scope as number of segments with inflammation evaluated as score 1 or more at aortic root and coronary arteries; $C$, severity score of each segment. Data are expressed as mean \pm SD of results from three individuals. ${ }^{*} P<0.05$ and ${ }^{* *} P<0.01$ (Student's $t$-test). 
A

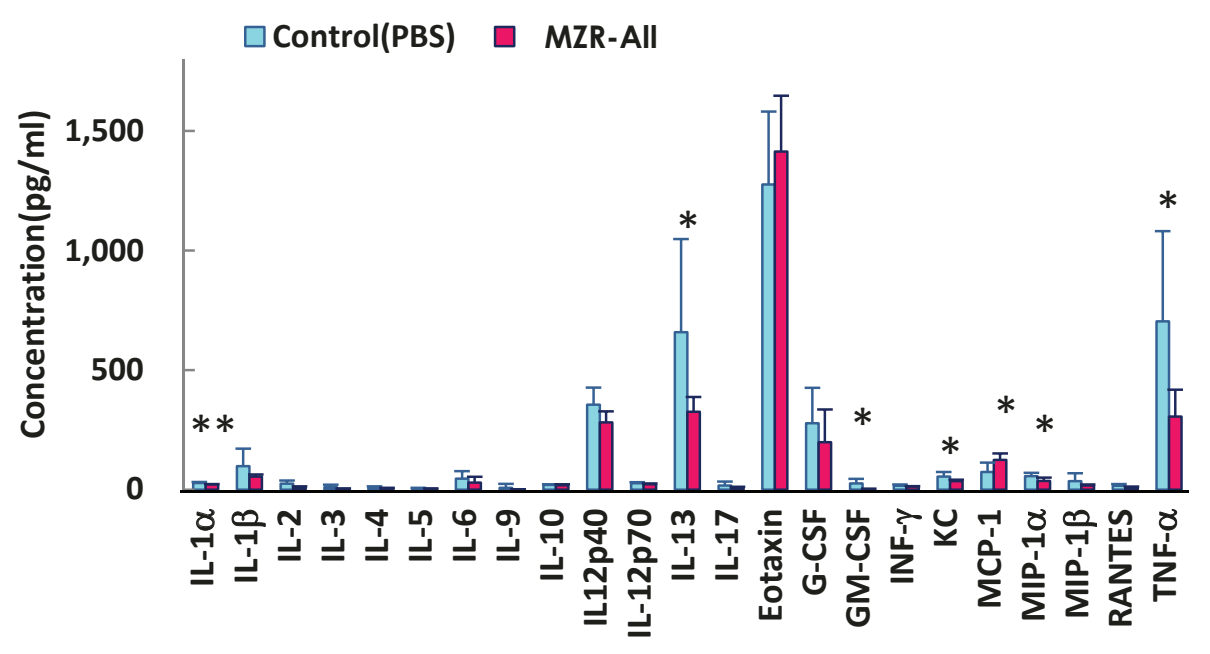

B

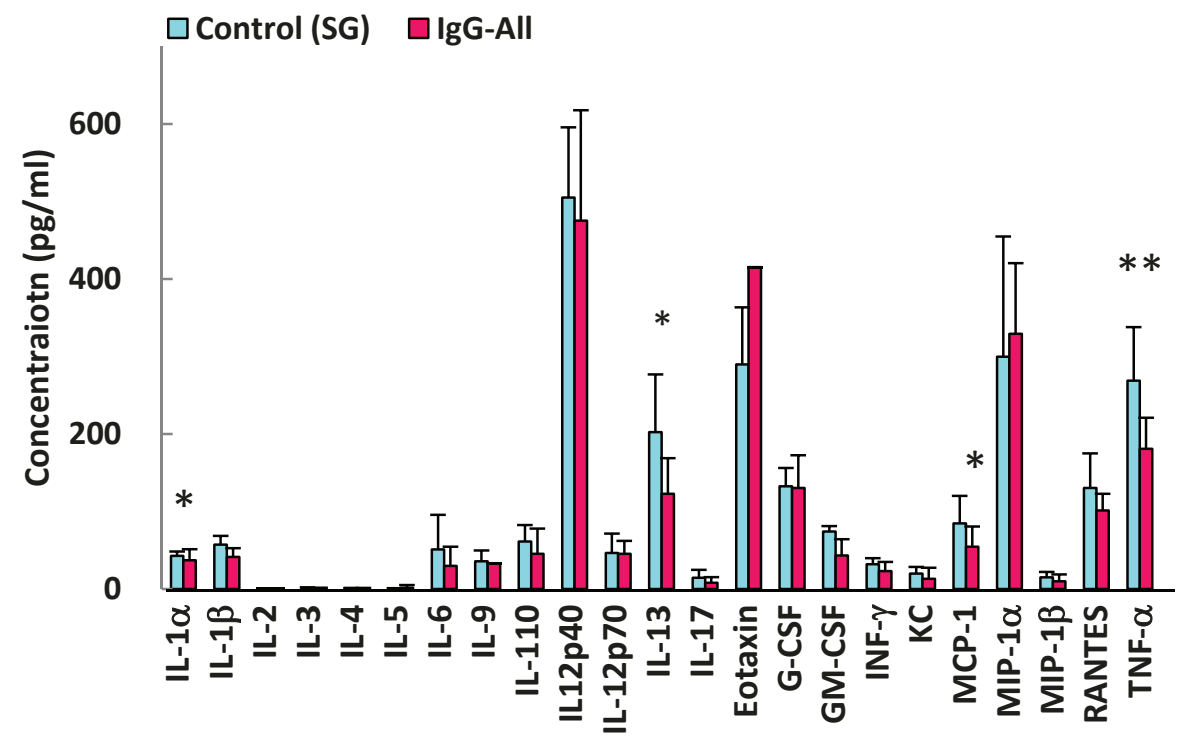

Figure 3 Reduction and enhancement of cytokines and chemokines by treatment with MZR and lgG. A, MZR treatment; B, IgG treatment. SG: saline including $0.1 \%$ glucose. Data are expressed as mean \pm SD of results from three individuals. ${ }^{*} P<0.05$ and ${ }^{* *} P<0.01$.

group, showing good response for suppression of the development of coronary arteritis (Figure 4B).

\section{Discussion}

Decrease of coronary arteritis by treatment with MZR

We here have shown the efficacy of MZR on vascular inflammation by using a KD vasculitis mouse model to develop alternative treatments for KD patients who are nonresponsive to IVIg treatment. The results here show that the incidence, scope, and degree of inflammation of the coronary arteries and the aortic root were suppressed by MZR administration. Coronary arteritis in this CAWS-induced vasculitis mouse model is also suppressed after administration of IVIg [33]. Furthermore, 


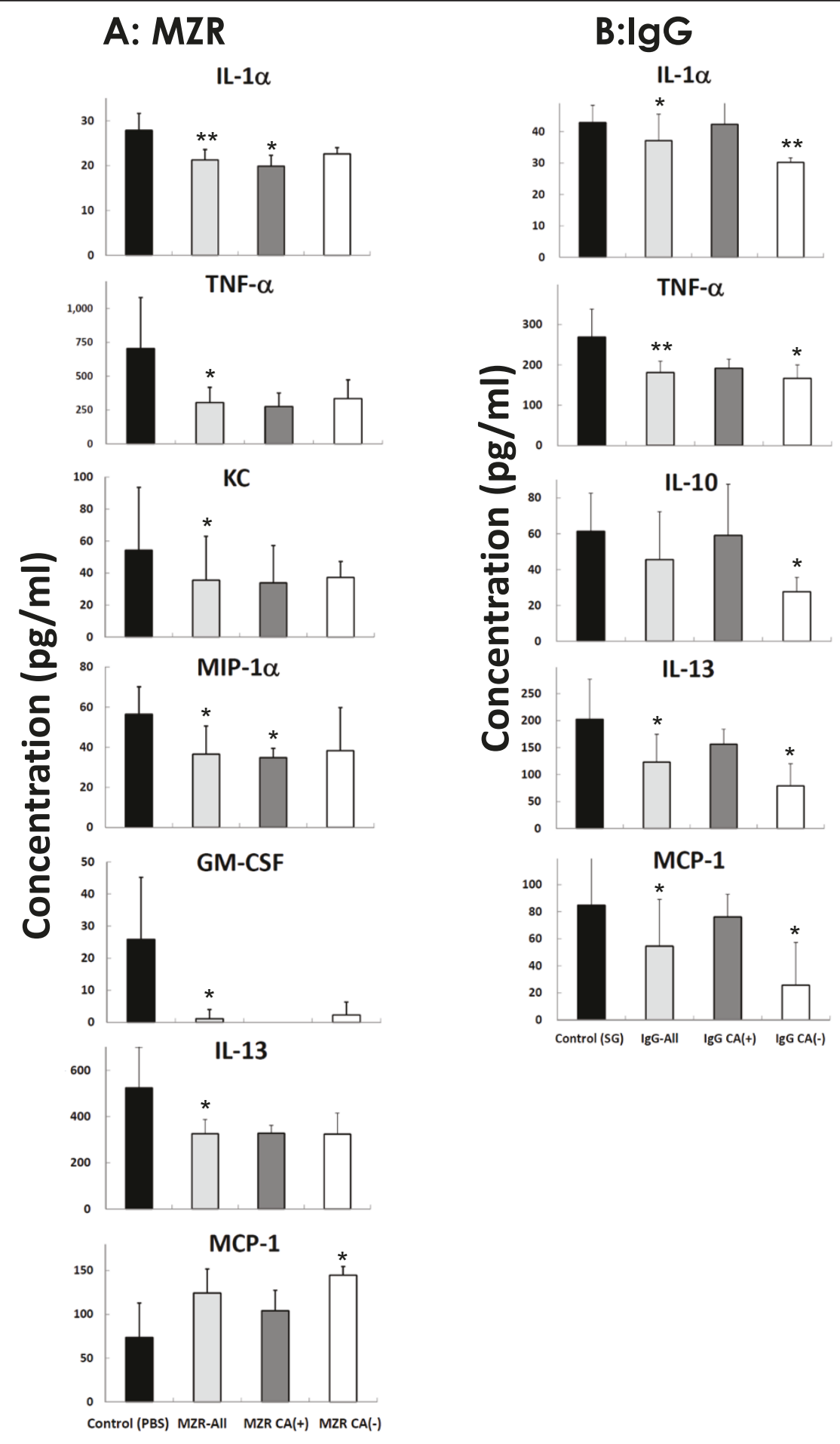

Figure 4 Reduction and enhancement of cytokines and chemokines in coronary arteritis treated with MZR and IgG. A, MZR treatment; $B$, IgG treatment. $C A(+)$ : coronary arteritis score $>0, C A(-)$ : coronary arteritis score $=0$, SG: saline including $0.1 \%$ glucose. Data are expressed as mean $\pm S D$ of results from three individuals. ${ }^{*} P<0.05$ and ${ }^{* *} P<0.01$. 
we have also demonstrated that the anti-TNF- $\alpha$ therapy that has been shown to be effective in treating some children unresponsive to IVIg therapy also dramatically suppresses the development of vasculitis in this mouse model of KD (manuscript in preparation). Thus this mouse model appears to be valuable for evaluation of alternative therapies for KD arteritis.

\section{Reduction of inflammatory cytokines and chemokines by treatment with MZR}

Some cytokines and chemokines such as IL-1 $\beta$, IL-2, sIL2R, IL-4, IL-6, IL-8, IL-10, IL-12, IL-15, RANTES, MCP-1, M-CSF, G-CSF, and MIPs are elevated in the blood of patients with acute-phase KD. Some elevated cytokines and chemokines are decreased by IVIg treatment in the acute phase, when it is effective [35]. On the other hand, IL-6, TNF- $\alpha$, IL-4, and IL-12 were increased in the plasma of the KD mouse model induced with $C$. albicans-derived substances (CADS) [36]. Moreover, IL-6 and IFN- $\gamma$ in splenocytes administered CAWS in C57BL/6 mice were elevated [37]. With IVIg treatment of KD model mice induced with CAWS, elevated proinflammatory cytokines IL-1 $\alpha$, TNF- $\alpha$, IL-10, IL-13, and MCP-1 were decreased in our data. Furthermore, chemokines IL-1 $\alpha$, TNF- $\alpha, \mathrm{KC}$, GM-CSF, IL-13, and MIP-1 $\alpha$ in plasma of autopsied mice were decreased in the MZR treatment group in the present study. The results with MZR treatement show similar effects as well as IgG treatment for KD model mice. However, supression levels of IL-1 $\alpha$, TNF- $\alpha$, IL-10, IL-13, and MIP- $1 \alpha$ in the recovery group from the coronary arteritis $(\mathrm{CA}(-))$ in the MZR group differed from those in the IgG group. Levels in the recovery group $(\mathrm{CA}(-))$ after MZR treatment were not suppressed, whereas those in the CA (-) group after IgG treatment were suppressed in the present study, which suggests that MZR may have a stronger effect than a high dose of IgG (400 mg/ $\mathrm{kg} /$ day for 5 days). Because these cytokines/chemokines decrease slightly after MZR treatment, they may have a role in the development of coronary arteritis in the KD model.

\section{Effective treatment with MZR of model mice for KD induced by CAWS}

In the present study, MZR treatment of the KD model mice significantly suppressed the development of coronary arteritis associated with significant suppression of levels of proinflamatory cytokines and chemokines in plasma. These results suggest association of the suppression of lymphocyte proliferation with MZR [38]. The mode of action of MZR is that it mainly blocks immunosuppression related to lymphocyte proliferation through inhibition of purine synthesis $[32,39,40]$. In the present study, the incidence of panarteritis decreased to half, and both the scope and severity of inflammation were limited after administration of MZR. In addition to the lymphocyte action, these observations suggest that MZR may act on functions of monocytes/macrophages and neutrophils, which are mainly involved in the development of inflammation, resulting in the possible suppression of coronary arteritis through suppression of proinflammatory cytokines and chemokines released from these cells. Indeed, recently, MZR acted to inhibit functions of lymphocytes as well as those of macrophages, such as migration and production of Nitrous Oxide Systems (NOS), IL- $1 \beta$, and TNF- $\alpha$ in a dosedependent manner $[41,42]$. Furthermore, in the mixed lymphocyte reaction method (MLR) of human peripheral blood mononuclear cells, the $\mathrm{IC}_{50}$ is $1 \mu \mathrm{g} / \mathrm{ml}$ [43]. In addition, MLR of T-cells in human peripheral blood, which are stimulated with anti-CD3 monoclonal antibody, shows an $\mathrm{IC}_{50}$ of less than $1 \mu \mathrm{g} / \mathrm{ml}$ for MZR and also phorbol myristate stimulation less than $5 \mu \mathrm{g} / \mathrm{ml}$ [44]. In addition, MZR also inhibits activation of M1 macrophages [42], which are classified as inflammatory, showing tissue injury and activation with IFN- $\gamma$. In the present study, suppression profiles of proinflammatory cytokines and chemokines by MZR treatment of KD model mice seem to be associated in the literature with those in the M1 macrophage. Therefore, the effect of MZR on KD model mice may be to inhibit the proliferation of lymphocytes and activation of macrophages and neutrophils associated with elevation of proinflamatory cytokines and chemokines.

Based on these observations, suppression of development of coronary arteritis associated with suppression of proinflammatory cytokines and chemokines by MZR treatment for the KD model mice suggests that MZR may be useful for patients with KD in the acute phase. MZR has been used as therapy for kidney transplantation, lupus nephritis, nephrotic syndrome, and rheumatoid disease with few side effects [24]. Furthermore, MZR has been used as maintenance treatment for ANCA-associated vasculitis, frequently relapsing nephrotic syndrome, and purpura nephritis $[29,30]$. Clinical use will be recommended for immune dysfunctions when the safety of long-time use becomes known. Therefore, MZR is a possible therapy for patients with KD who are nonresponsive to IVIg.

\section{Conclusions}

MZR treatment suppressed not only the incidence, range, and degree of vasculitis, but also inflammatory cytokines and chemokines in the plasma of the KD vasculitis model mice. It appears likely that MZR may prove to be a useful for alternative treatment for KD.

\section{Abbreviations used}

ANCA: anti-neutrophil cytoplasmic autoantibody; CAWS: Candida albicans water-soluble fractions; H\&E: 
hematoxylin and eosin; IVIg: intravenous immunoglobulin; KD: Kawasaki disease; MLR: mixed lymphocytes reaction method; MZR: mizoribine; NOS: Nitrous Oxide Systems: PBS; Dulbecco's phosphate- buffered saline; SG: saline containing $0.1 \%$ glucose.

\section{Contribution of authors}

KT: Histological evaluations of coronary arteritis. TO: Histological evaluations of coronary arteritis. TN: Measurement and analysis of cytokines and chemokines. YY: Measurement and analysis of cytokines and chemokines. HY: Histological evaluations of coronary arteritis. NNM: Preparation of CAWS. NO: Preparation of CAWS. TS: Planning treatments with MZR and IgG, and clinical evaluation. TO: Planning treatments with MZR and IgG, and clinical evaluation. KS: Measurement and analysis of cytokines and chemokines, correspondence to all evaluation of this study. All authors read and approved the final manuscript.

\section{Acknowledgements}

We thank Dr. Shiro Naoe (Department of Biomedical Engineering, Toin University of Yokohama, Yokohama). We are also grateful to Mr. Kazuo Tomizawa of NIID for excellent assistance with animal experiments.

\section{Author details}

'Department of Pathology, Toho University Ohashi Medical Center, Meguroku, Tokyo, 153-8515, Japan. ${ }^{2}$ Inflammation Program, Dept. of Immunology, Chiba University Graduate School of Medicine, Chuo-ku, Chiba, 260-8670, Japan. ${ }^{3}$ Laboratory for Immunopharmacology of Microbial Products, School of Pharmacy, Tokyo University of Pharmacy and Life Science, Hachioji, Tokyo 192-0392, Japan. ${ }^{4}$ Department of Pediatrics, Toho University Omori Medical Center, Ota-ku, Tokyo, 143-8541, Japan. ${ }^{5}$ Kure Kyosai Hospital, Kure, Hiroshima, 737-8505, Japan.

\section{Competing interests}

The authors declare that they have no competing interests.

Received: 3 April 2011 Accepted: 29 September 2011 Published: 29 September 2011

\section{References}

1. Kawasaki T: Acute febrile muco-cutaneous lymph node syndrome in young children with unique digital desquamation. Jpn J Allergol 1967, 16:178-222.

2. Kawasaki T, Kosaki F, Okawa S, Shigematsu I, Yanagawa H: New infantile acute febrile mucocutaneous lymph node syndrome (MLNS) prevailing in Japan. Pediatrics 1974, 54:271-276.

3. Furusho K, Sato K, Soeda T, Matsumoto H, Okabe T, Hirota T, Kawada S: High-dose intravenous gammaglobulin for Kawasaki disease. Lancet 1983, 10(8363):1359.

4. Nakamura Y, Yashiro M, Uehara R, Sadakane A, Chihara I, Aoyama Y, Kotani $\mathrm{K}$, Yanagawa H: Epidemiologic features of Kawasaki disease in Japan: results of the 2007-2008 nationwide survey. J Epidemiol 2010, 20:302-307.

5. Sundel RP, Burns JC, Baker A, Beiser AS, Newburger JW: Gamma globulin re-treatment in Kawasaki disease. J Pediatr 1993, 123:657-659.

6. Shinohara M, Sone K, Tomomasa T, Morikawa A: Corticosteroids in the treatment of the acute phase of Kawasaki disease. J Pediatr 1999, 135:465-469.

7. Kobayashi T, Inoue Y, Takeuchi K, Okada Y, Tamura K, Tomomasa T, Kobayashi T, Morikawa A: Prediction of intravenous immunoglobulin unresponsiveness in patients with Kawasaki disease. Circulation 2006, $113: 2606-2612$
8. Villain E, Kachaner J, Sidi D, Blaysat G, Piéchaud JF, Pedroni E: Trial of prevention of coronary aneurysm in Kawasaki's disease using plasma exchange or infusion of immunoglobulins. Arch Fr Pediatr 1987, 44:79-83.

9. Imagawa T, Mori M, Miyamae T, Ito S, Nakamura T, Yasui K, Kimura H, Yokota S: Plasma exchange for refractory Kawasaki disease. Eur J Pediatr 2004, 163:263-264

10. Mori M, Imagawa T, Katakura S, Miyamae T, Okuyama K, Ito S, Nakamura T, Kimura $\mathrm{H}$, Yokota S: Efficacy of plasma exchange of plasma exchange therapy for Kawasaki disease intractable to intravenous gamma-globulin. Mod Rheumatol 2004, 14:43-47.

11. Wright DA, Newburger JW, Baker A, Sundel RP: Treatment of immune globulin-resistant Kawasaki disease with pulsed doses of corticosteroids. J Pediatr 1996, 128:146-149

12. Wallace CA, French JW, Kahn SJ, Sherry DD: Initial intravenous gammaglobulin treatment failure in Kawasaki disease. Pediatrics 2000, 105:E78

13. Zaitsu M, Hamasaki Y, Tashiro K, Matsuo M, Ichimaru T, Fujita I, Tasaki H, Miyazaki S: Ulinastatin, an elastase inhibitor, inhibits the increased mRNA expression of prostaglandin $\mathrm{H} 2$ synthase-type 2 in Kawasaki disease. J Infect Dis 2000, 181:1101-1109.

14. Miura M, Ohki H, Tsuchihashi T, Yamagishi H, Katada Y, Yamada K, Yamashita Y, Sugaya A, Komiyama O, Shiro H: Coronary risk factors in Kawasaki disease treated with additional gammaglobulin. Arch Dis Child 2004, 89:776-780.

15. Uehara R, Yashiro M, Oki I, Nakamura Y, Yanagawa H: Re-treatment regimens for acute stage of Kawasaki disease patients who failed to respond to initial intravenous immunoglobulin therapy: analysis from the 17th nationwide survey. Pediatr Int 2007, 49:427-430

16. Iwashima S, Seguchi M, Matubayashi T, Ohzeki T: Ulinastatin therapy in Kawasaki disease. Clin Drug Investig 2007, 27:691-696.

17. Raman V, Kim J, Sharkey A, Chatila T: Response of refractory Kawasaki disease to pulse steroid and cyclosporine A therapy. Pediatr Infect Dis 2001, 20:635-637.

18. Adachi S, Sakaguchi H, Kuwahara T, Uchida Y, Fukao T, Kondo N: High regression rate of coronary aneurysms developed in patients with immune globulin. Tohoku J Exp Med 2010, 220:285-290.

19. Weiss JE, Eberhard BA, Chowdhury D, Gottlieb BS: Infliximab as a novel therapy for refractory Kawasaki disease. J Rheumatol 2004, 31:808-810.

20. Saji T, Kemmotsu Y: Infliximab for Kawasaki syndrome [reply]. J Pediat 2006, 149:426.

21. Burns JC, Best BM, Mejias A, Mahony L, Fixler DE, Jafri HS, Melish ME, Jackson MA, Asmar BI, Lang DJ, Connor JD, Capparelli EV, Keen ML, Mamun K, Keenan GF, Ramilo O: Infliximab treatment of intravenous immunoglobulin-resistant Kawasaki disease. J Pediatr 2008, 153:833-838.

22. Shirley DA, Stephens I: Primary treatment of incomplete Kawasaki disease with infliximab and methylprednisolone in a patient with a contraindication to intravenous immune globulin. Pediatr Infect Dis $J$ 2010, 29:978-979.

23. Son MB, Gauvreau K, Burns JC, Corinaldesi E, Tremoulet AH, Watson VE, Baker A, Fulton DR, Sundel RP, Newburger JW: Infliximab for intravenous immunoglobulin resistance in Kawasaki disease: a retrospective study. $J$ Pediatr 2011, 158:644-649.

24. Ishikawa H: Mizoribine and mycophenolate mofetil. Curr Medicinal Chem 1999, 6:575-597.

25. Yokota S: Mizoribine: Mode of action and effects in clinical use. Pediatr Int 2002, 44:196-198.

26. Kawasaki Y, Hosoya M, Suzuki J, Onishi N, Takahashi A, Isome M, Nozawa R, Suzuki $\mathrm{H}$ : Efficacy of multidrug therapy combined with mizoribine in children with diffuse IgA nephropathy in comparison with multidrug therapy without mizoribine and with methylprednisolone pulse therapy. Am J Nephrol 2004, 24:576-581.

27. Yoshikawa N, Nakanishi K, Ishikura K, Hataya H, lijima K, Honda M: Japanese Pediatric IgA Nephropathy Treatment Study Group: combination therapy with mizoribine for severe childhood IgA nephropathy: a pilot study. Pediatr Nephrol 2008, 23:757-763.

28. Honda M: Nephrotic syndrome and mizoribine in children. Pediatr Int 2002, 44:210-216.

29. Hirayama K, Kobayashi M, Hashimoto Y, Usui J, Shimizu Y, Hirayama A, Yoh K, Yamagata K, Nagase S, Nagata M, Koyama A: Treatment with the purine synthesis inhibitor mizoribine for ANCA-associated renal vasculitis. Am J Kidney Dis 2004, 44:57-63. 
30. Ohtomo Y, Fujinaga S, Takada M, Murakami H, Akashi S, Shimizu T, Kaneko K, Yamashiro Y: High-dose mizoribine therapy for childhood-onset frequently relapsing steroid-dependent nephritic syndrome with cyclosporine nephrotoxicity. Pediatr Nephrol 2005, 20:1744-1749.

31. Takahashi K, Oharaseki T, Wakayama M, Yokouchi Y, Naoe S, Murata H: Histopathological features of murine systemic vasculitis caused by Candida albicans extract - an animal model of Kawasaki disease. Inflamm Res 2004, 53:72-77.

32. Okubo M, Chen XM, Kamata K, Masaki Y, Uchiyama T: Suppressive effect of mizoribine on humoral antibody production in DBA/2 mice. Transplantation 1986, 41:495-498.

33. Takahashi K, Oharaseki T, Nagai-Miura N, Ohno N, Ishida-Okawara A, Yamada H, Kaneshiro Y, Naoe S, Suzuki K: Administration of human immunoglobulin inhibited development of vasculitis in a murine model of vasculitis induced with CAWS, Candida albicans water soluble fraction. Modern Reumatol 2010, 20:160-167.

34. Tomizawa K, Nagao T, Kusunoki R, Saiga K, Oshima M, Kobayashi K, Nakayama T, Tanokura M, Suzuki K: Reduction of MPO-ANCA epitopes in SCG/Kj mice by 15 -deoxyspergualin treatment restricted by lgG2b associated with crescentic glomerulonephritis. Rheumatology (Oxford) 2010, 49:1245-1256.

35. Jibiki T, Terai M, Kohno Y: High concentrations of interleukin-8 and monocyte chemoattractant protein-1 in urine of patients with acute Kawasaki disease. Eur J Pediatr 2004, 163:749-750.

36. Oharaseki T, Kameoka Y, Kura F, Persad AS, Suzuki K, Naoe S: Susceptibility loci to coronary arteritis in animal model of Kawasaki disease induced with Candida albicans-derived substances. Microbiol Immunol 2005, 49:181-189.

37. Nagi-Miura N, Shingo Y, Adachi Y, Ishida-Okawara A, Oharaseki T, Takahashi K, Naoe S, Suzuki K, Ohno N: Induction of coronary arteritis with administration of CAWS (Candida albicans water-soluble fraction) depending on mouse strains. Immunopharmacol Immunotoxicol 2004, 26:527-543.

38. Koyama H, Tsuji M: Genetic and biochemical studies on the activation and cytotoxic mechanism of bredinine, a potent inhibitor of purine biosynthesis in mammalian cells. Biochem Pharmacol 1983, 32:3547-3553.

39. Gan L, Mohammad R, Seyedsayamdost, Shuto S, Matsuda A, Gregory A, Hedstrom PL: The immunosuppressive agent mizoribine monophosphate forms a transition state analogue complex with inosine monophosphete dehydrogenase. Biochemistry 2003, 42:857-863.

40. Kusumi T, Tsuda M, Katsunuma T, Yamamura M: Dual inhibitory effect of bredinin. Cell Biochem Func 1988, 7:201-204.

41. Kikuchi Y, Imakiire T, Yamada M, Saigusa T, Hyodo T, Hyodo N, Suzuki S, Miura S: Mizoribine reduces renal injury and macrophage infiltration in non-insulin-dependent diabetic rats. Nephrol Dial Transplant 2005, 20:1573-1581.

42. Ikezumi Y, Suzuki T, Karasawa T, Hasegawa H, Kawachi H, NikolicPaterson DJ, Uchiyama M: Contrasting effects of steroids and mizoribine on macrophage activation and glomerular lesions in rat Thy-1 mesangial proliferative glomerulonephritis. Am J Nephrol 2010, 31:273-282.

43. Sonda K, Takahashi K, Tanabe K, Funchinoue S, Hayasaka Y, Kawaguchi H, Teraoka S, Toma H: Clinical pharmacokinetic study of mizoribine in renal transplantation patients. Transplant Proc 1996, 28:3643-3648.

44. Turka LA, Dayton J, Sinclair G, Thompson CB, Mitchell BS: Guanine ribonucleotide depletion inhibits T cell activation. J Clin Invest 1991, 87:940-948.

doi:10.1186/1546-0096-9-30

Cite this article as: Takahashi et al: Mizoribine provides effective treatment of sequential histological change of arteritis and reduction of inflammatory cytokines and chemokines in an animal model of Kawasaki disease. Pediatric Rheumatology 2011 9:30.

\section{Submit your next manuscript to BioMed Central and take full advantage of:}

- Convenient online submission

- Thorough peer review

- No space constraints or color figure charges

- Immediate publication on acceptance

- Inclusion in PubMed, CAS, Scopus and Google Scholar

- Research which is freely available for redistribution

Submit your manuscript at www.biomedcentral.com/submit 